enterology, as well as two European and one International Society devoted entirely to the oesophagus. The substantial involvement by surgeons in these developments is reflected in this large work comprising 105 chapters in almost 1000 pages. Surgery of the oesophagus is edited by Professor Glyn Jamieson, an accomplished oesophageal surgeon from Adelaide. He has undertaken the Herculean task of contributing extensively to this large volume and editing contributions from 124 experts from various parts of the world. Although primarily intended for oesophageal surgeons, there is much in the book for all with an interest in the oesophagus, with excellent sections on anatomy and physiology, investigation and pathophysiology of oesophageal disease. Neither is the book written entirely by surgeons; well respected gastroenterologists including Castell and Dent join with pathologists, radiologists and oncologists, emphasising the importance of interdisciplinary collaboration in the investigation and management of oesophageal discase.

The principal aim of the book, however, is an extremely comprehensive coverage of all aspects of oesophageal surgery, with greater emphasis on technical detail than any previous volume, and it achieves these objectives admirably. Inevitably, an undertaking of this size results in some repetition and occasional loss of cohesion. The paucity of detail on ambulatory $\mathrm{pH}$ monitoring and virtual absence of objective data in the 17 chapters on the various surgical approaches to resistant gastro-oesophageal reflux and its complications is disappointing and presumably reflects the considerable time in compiling a work of this magnitude, during which many advances in these fields have occurred.

Surgery of the oesophagus is a comprehensive reference work which contains much to interest all who have a significant involvement in the management of patients with oesophageal disease.

A WATSON

Gastric protection. Edited by R Cheli. (Pp. 300; illustrated; $£ 107 \cdot 50$.) New York: Raven Press, 1988. This book contains 22 chapters each averaging 12 pages in length concerned with gastric protection. It is divided into four sections - physiological concepts, aggressive factors, anti-secretory drugs and protective drugs. The bulk of the references run up to and including 1986 and there are a reasonable total quantity. The figures and diagrams are generally clear and relevant.

Although the volume covers a wide range of subjects, it is generally rather short on critical analysis and there tends to be a scientific switchback as apparently conflicting data sets or opinions are placed opposite each other without attempts to reconcile or resolve. The reader therefore obtains sets of possibilities but is short on syntheses. The clinical reader will also find that much is devoted to experimental studies and clinical relevance is rather limited. The chapter on food allergy is a case in point which in essence lists a variety of mechanisms and medications without clarifying their individual importance or giving clinical guidance in dealing with affected individuals. Similar considerations apply to the chapter on ethanol induced damage with a list of mechanisms but no consideration of the clinical problems arising. Elsewhere one can question individual generalisations thus, is the measurement of the capability of fresh tissue to generate prostaglandins 'convenient and reliable'. Much published evidence suggests otherwise.

This book is a useful reference source and is helpful in summarising sets of concepts. The reader seeking a critical synthesis will, however, need to look elsewhere.

M J S I.ANGMAN

Year book of digestive diseases, 1988 . Edited by $\mathrm{N} \mathrm{J}$ Greenberger, and F G Moody. (Pp. 475; illustrated; £33.50.) London: Wolfe Medical, 1988.

Previous editions of this book have been favourably reviewed in these columns. It is, therefore, sufficient to point out that these apparently inexhaustible editors have once again maintained their high standards of critical selection and comment and that this work remains, for this reviewer, the best of the annual review publications for gastroenterologists.

\section{DAVID WINGATE}

Viral hepatitis and liver disease. By A $\mathrm{J}$ Zuckerman. (Pp. 1136; illustrated; price not stated.) New York: Liss, 1988.

This enormous volume of more than 1100 pages represents the proceedings of an International Symposium on Viral Hepatitis and Liver Disease held in London in May 1987. The 368 articles describe the results of the latest research being carried out by various groups throughout the world. There are sections on hepatitis $A$ including some early results with hepatitis $A$ vaccines, enteric and parenteral non-A non-B hepatitis, molecular biological aspects of hepatitis B virus, delta hepatitis and animal hepatitis viruses. There are also sections on immunology of viral hepatitis, hepatocellular carcinoma and the treatment of viral hepatitis including antiviral agents and liver transplantation for fulminant hepatitis while the work concludes with the latest results on hepatitis $B$ vaccines. 